\section{Sarcoma or Phaeochromocytoma? Lessons from multi-disciplinary team working}

\author{
Paul Grant,1 Omar Mustafa, 1 \\ Val Lewington,2 John Quin'1 \\ 1Department of Diabetes and \\ Endocrinology, Royal Sussex County \\ Hospital; 2Department of Nuclear \\ Medicine, Royal Marsden Hospital, UK
}

\begin{abstract}
An issue in the practice of oncology is the patient with a rare malignancy, where the challenge to the clinician is to rapidly understand the diagnosis and prioritise the best management approach. We present the case of a patient who was initially thought to have a malignant sarcoma and was eventually found to have an unusual, aggressive neuroendocrine tumour. This case stresses the importance of multi-disciplinary team (MDT) working and good communication and raises the pertinent issue of risk analysis in such settings. The case has greater relevance as it has led to a re-evaluation of how such cases are handled and heralded the introduction of a system of internal peer review.
\end{abstract}

\section{Case Report}

A 51-year-old woman was referred to the colo-rectal clinic due to anaemia, abdominal discomfort and the discovery of a left sided abdominal mass. Her medical history included uncontrolled hypertension and she had been previously seen in the chest pain clinic for attacks of atypical chest pains, which were put down to gastro-oesophageal reflux disease.

She was found to have a Haemoglobin of 6.8 $\mathrm{g} / \mathrm{dL}$, platelets of 700 and an ESR of 100 . An abdominal CT scan was arranged to investigate her abdominal pain (Figure 1) which revealed a large $10 \mathrm{~cm}$ intra-abdominal mass with gross left sided hydronephrosis due to renal obstruction, appearing to originate from the ileo-psoas muscle and the radiological diagnosis of a sarcoma was made on clinical grounds.

The patient was initially informed that she had a malignant sarcoma with a poor prognosis. She went on to have a whole body staging CT and was subsequently discussed at a Sarcoma multi-disciplinary team meeting (MDM) at a specialist oncology centre. The CT scan identified a suspicious lesion in the T11 vertebrae and she went on to have an isotope bone scan confirming a vertebral metastasis and likely metastatic deposits in both scapulae. No differential diagnoses were discussed at this point and there was a delay in getting the patient re-reviewed at the MDM as it was felt that little could be offered treatment wise.

Further review was requested by the radiology specialist as this was considered unlikely characteristic behaviour for a sarcoma, alternative diagnoses were considered at that point and further investigations arranged. She had tru-cut core biopsies which were reported as showing a left sided, retroperitoneal metastatic paraganglioma. This was unexpected. At the suggestion of a nuclear medicine specialist she went on to have $24 \mathrm{~h}$ urinary catecholamines which were significantly elevated with Noradrenaline levels of 6299 (units) and Dopamine 6278 (units) and an MIBG scan which was consistent with the diagnosis.

She was referred on to Urology for stenting of her left ureter and then to the Endocrinology department (via the neuro-endocrine MDT) for peri-operative management. She required alpha-blockade with phenoxybenzamine, then beta-blockade to control her hypertension.

The patient underwent a surgical resection of a $15 \mathrm{~cm}$ periaortic tumour with en-bloc left nephrectomy, retroperitonectomy, sigmoid resection and resection of the left crus of the diaphragm. The patient made a good post operative recovery and subsequently proceeded to high activity I-131 mIBG therapy to treat the known skeletal metastases.

\section{Discussion}

Many cases in endocrinology are best discussed in a MDT fashion. This means that important management decisions are not taken by a single doctor, but discussed with a group of experts from different disciplines including oncology, radiology, pathology, specialist surgery and specialist nurses. A formal regular meeting is required to facilitate this. ${ }^{1}$

This case of a malignant paraganglioma demonstrates an important theme that is easy to observe in retrospect when managing neuroendocrine tumours. There may be a prolonged history of vague, non-specific signs and symptoms that present to different specialities prior to diagnosis. Uncontrolled hypertension (especially in younger patients) should raise suspicion.

Paragangliomas are histologically identical to phaeochromocytomas that arise from the adrenal medulla. Malignancy is more common in paragangliomas $(30-50 \%)$ than in phaeochromocytomas (10-15\%), with local invasion, destruction of adjacent vertebrae, and distant metastases to lungs, lymph nodes,
Correspondence: Paul Grant, Royal Sussex County Hospital, Eastern Road, Brighton

East Sussex, BN2 5UD, UK

Telefax: +44.01273.696955.

E-mail: drpaul.grant@doctors.org.uk

Key words: sarcoma, phaeochromocytoma, multidisciplinary team, working.

Acknowledgments: many thanks to Mr. Klaus Martin-Schulte.

Conflict of interest: the authors report no conflicts of interest.

Received for publication: 9 July 2011.

Revision received: 13 September 2011.

Accepted for publication: 24 September 2011.

This work is licensed under a Creative Commons Attribution NonCommercial 3.0 License (CC BYNC 3.0).

(C) Copyright P. Grant et al., 2011

Licensee PAGEPress, Italy

Endocrinology Studies 2011; 1:e10

doi:10.4081/es.2011.e10

and bones. ${ }^{2}$ Extra-adrenal phaeochromocytomas are rare and unusual. They can present in many different ways and the risk of malignancy is high. Although all paragangliomas contain neurosecretory granules, only about 1$3 \%$ have evidence of hormonal hypersecretion. The main treatment modalities are surgery, embolisation, combination chemotherapy and targeted radionuclide therapy. ${ }^{3}$

This woman's cancer was initially labelled as an inoperable malignant tumour and the patient was informed of this accordingly. There was no discussion of alternative diagnoses and no risk analysis had been undertaken to confirm this. Paragangliomas, which may be more amenable to effective treatment can present in similar ways to sarcoma and so must be considered in the differential before the patient is given the diagnosis.

Multidisciplinary team meetings have been shown to be beneficial in the management of patients with cancer. UK Government recommendations introduced in 2002 suggested that more meetings were needed to take place to optimise cancer care in the UK. ${ }^{4}$ Multidisciplinary teams are an important way of managing complex cases but they are not always perfect. Themes which emerge from the literature suggest that there are benefits and problems with MDT's. ${ }^{5}$ The advantages of team working include; information gathering, improved patient care, planning and decision reassurance. Problems identified included time constraints, excessive radiological workload, miscommunication, cost implications and the loss of nurse led meetings. There is 
commonly little contribution by certain allied heath professionals who find the atmosphere generated by medical staff combative.

In light of our case and other recent research, ${ }^{4-7}$ we feel that there are important lessons to be learnt: i) radiological diagnosis does not always translate to histological diagnosis; ii) prognosis should ideally be discussed following tissue diagnosis; iii) cross communication between MDT's is crucial to solving complex cases; iv) communication within the group should be free and equitable.

Following the above case, it was agreed that

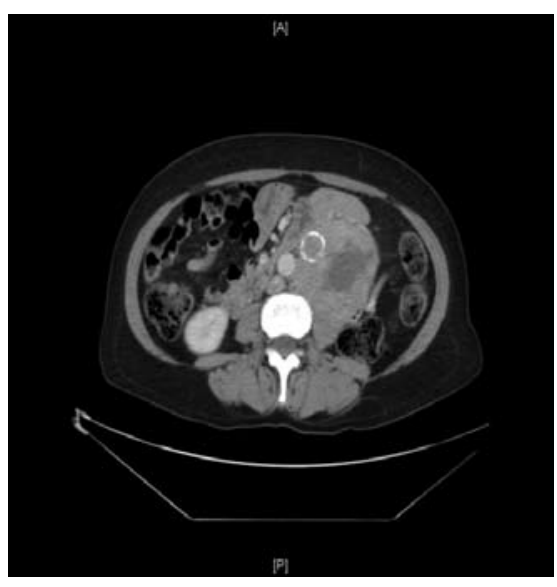

Figure 1. Abdominal computed tomography scan demonstrates a large mass in the retroperitoneum to the left of the midline with cystic/necrotic elements and some calcification. the pathway for both solid malignancy and neuro-endocrine referrals be reconsidered and an element of risk analysis / internal evaluation be developed to avoid errors, especially in two difficult areas of exploring differential diagnoses and subsequently agreeing on a management plan. The department in question has now also undergone an external peer review by visitation from the UK's Society for Endocrinology which has made some recommendations for improving multi-disciplinary working. ${ }^{8}$ The local institutional committee has also acknowledged that there needs to be greater cross communication between different MDT's. For example, a pituitary MDT may have overlapping aims and objectives or even competing management goals with a neurooncology MDT which may be dealing with the same case.

Our case therefore highlights two important factors, firstly that rare malignancies need to be dealt with in specialist, experienced centres and secondly that MDT's whilst a beneficial approach, need to have a rigorous element of quality control. ${ }^{9}$

\section{References}

1. Wagholikar A, Fung M, Nelson C. A pilot study on understanding the journey of advanced prostate cancer patients. Stud Health Technol Inform 2011;168:165-71.

2. Loh KC, Fitzgerald PA, Matthay KK et al.
The treatment of malignant pheochromocytoma with iodine-131 metaiodobenzylgua-nidine (131I-MIBG): a comprehensive review of 116 reported patients. J Endocrinol Invest 1997;20:648-58.

3. Roman S. Pheochromocytoma and functional paraganglioma. Curr Opin Oncol 2004;16:8-12.

4. Arya AK. The function of the multidisciplinary team meeting for head and neck cancer: A qualitative analysis. University of Chester. Available from: http://chesterrep. openrepository.com/cdr/handle/10034/ 79894

5. Saini KS, Taylor C, Ramirez AJ. The Role of the multidisciplinary team in breast cancer management: results form a large international survey involving 39 countries. Ann Oncol 2011 Aug 4 [Epub ahead of print].

6. Acher PL, Young AJ, Etherington-Foy R et al. Improving Outcomes in Urological Cancers: the impact of multidisciplinary team meetings. Int J Surg 200;3:121-3.

7. Blazeby JM, Wilson L, Metcalfe C et al. Analysis of Clinical Decision-making in Multi-disciplinary cancer teams. Ann Oncol 2006;17:457-60.

8. Society for Endocrinology Peer Review. Available from: www.endocrinology.org/ about/projects/peerreview

9. Grant P. Performance Management in Clinical Endocrinology. Int $\mathrm{J}$ Clin Leadership 2011;17:83-5. 